\title{
Parasitic Events in Envelope Analysis
}

\section{J. Doubek, M. Kreidl}

Envelope analysis allows fast fault location of individual gearboxes and parts of bearings by repetition frequency determination of the mechanical catch of an amplitude-modulated signal. Systematic faults arise when using envelope analysis on a signal with strong changes. The source of these events is the range of function definition of $1 / \pi t$ used in convolution integral definition. This integral is used for Hilbert image calculation of analyzed signal. Overshoots (almost similar to Gibbs events on a synthetic signal using the Fourier series) are result from these faults. Overshoots are caused by parasitic spectral lines in the frequency domain, which can produce faulty diagnostic analysis.

This paper describes systematic arising during faults rising by signal numerical calculation using envelope analysis with Hilbert transform. It goes on to offer a mathematical analysis of these systematic faults.

Keywords: gearbox, bearing, envelope analysis, Hilbert transform, parasitic spectral lines.

\section{Envelope analysis}

Envelope analysis is deeply connected to the Hilbert transform [1], [5]. The Hilbert transform of signal $x(t)$ is defined by the following equation

$$
\widetilde{x}(t)=H\{x(t)\}=\frac{1}{\pi} \int_{-\infty}^{\infty} \frac{x(\tau)}{t-\tau} \mathrm{d} \tau
$$

where $\widetilde{x}(t)$ is Hilbert image of signal $x(t)$, also refered to as the quadrature part to signal $x(t)$.

The inverse Hilbert transform is defined by

$$
x(t)=H^{-1}\{\widetilde{x}(t)\}=-\frac{1}{\pi} \int_{-\infty}^{\infty} \frac{\widetilde{x}(\tau)}{t-\tau} \mathrm{d} \tau .
$$

Using the definition of a convolution we can define the Hilbert transform by

$$
\begin{aligned}
& \widetilde{x}(t)=\frac{1}{\pi t} * x(t) \\
& x(t)=-\frac{1}{\pi t} * \widetilde{x}(t) .
\end{aligned}
$$

The complex signal $\psi(\mathrm{t})$ whose imaginary part $\widetilde{x}(t)$ is the Hilbert transform of the real part $x(t)$ is called the analytic signal.

$$
\psi(t)=x(t)+j \widetilde{x}(t)
$$

where $\widetilde{x}(t)=H\{x(t)\}$.

The analytical signal as a complex function in the time domain can be expressed as a complex function in Euler form by

$$
\psi(t)=E(t) e^{-j \beta(t)},
$$

where is $E(t)$ is the amplitude of a complex function in the time domain,

$\beta(t)$ is the phase shift of a complex function in the time domain.

The following equations are valid for $E(t)$ and $\beta(t)$ :

$$
\begin{aligned}
& E(t)=\sqrt{x^{2}(t)+\tilde{x}^{2}(t)} \\
& \beta(t)=\operatorname{atan} \frac{\tilde{x}(t)}{x(t)} .
\end{aligned}
$$

Function $E(t)$ is called a signal $x(t)$ envelope.

The following equations are valid for the Hilbert transform of a harmonic signal:

$$
\begin{aligned}
& H\{\cos (\omega t+\varphi)\}=\sin (\omega t+\varphi) \\
& H\{\sin (\omega t+\varphi)\}=-\cos (\omega t+\varphi) .
\end{aligned}
$$

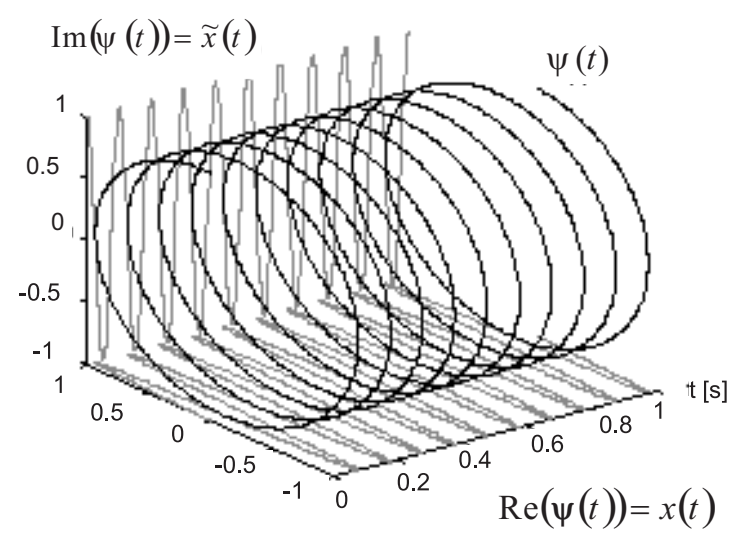

a)

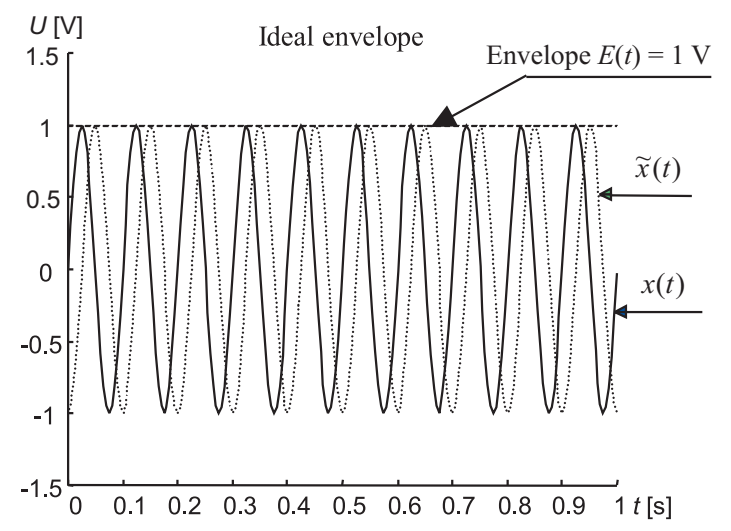

b)

Fig. 1: Analytical signal and envelope of $x(t)=\sin (2 \pi 10 t+\varphi)$, $\widetilde{x}(t)=\cos (2 \pi 10 t+\varphi), \varphi=0$ 
These equations show that the envelope $E(t)$ of the harmonic signal $x(t)=\sin (\omega t)$ is equal to:

$$
E(t)=\sqrt{x^{2}(t)+\widetilde{x}^{2}(t)}=\sqrt{\sin (\omega t)^{2}+\cos (\omega t)^{2}}=1 .
$$

The analytical signal and envelope is shown in Fig. 1.

\section{Envelope analysis faults}

Fig. 1 shows a part of harmonic signal $x(t)$, its analytically calculated quadrature part $\widetilde{x}(t)$ and envelope $E(t)$. Figure la shows analytical signal $\psi(t)$ in complex space. Figure $1 \mathrm{~b}$ shows the same signal in two dimensions.

\subsection{The edge measurement interval effect on a harmonic signal envelope}

The envelope of a harmonic signal is shown in Figure 2a. The quadrature part was obtained by a numerical calculation of the analytical signal in comparison to an analytical calculation (see Figure 1b). The envelope distortion at the edge of the measurement interval is shown in Figure 2a. Figure 2b shows the same signal but with a phase shift of $\varphi=\pi / 2$. Figure 2a shows a $50 \%$ deflection of the envelope in comparison to the ideal. Figure $2 \mathrm{~b}$ shows a $70 \%$ deflection of the envelope in comparison to the ideal.

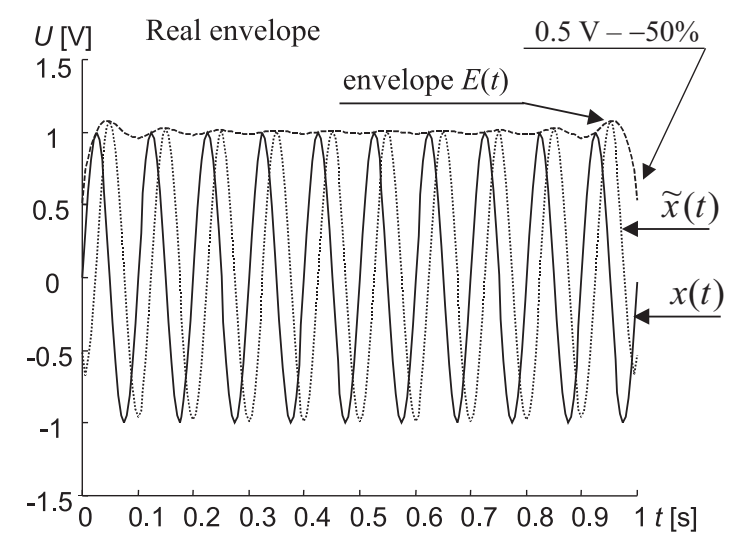

a)

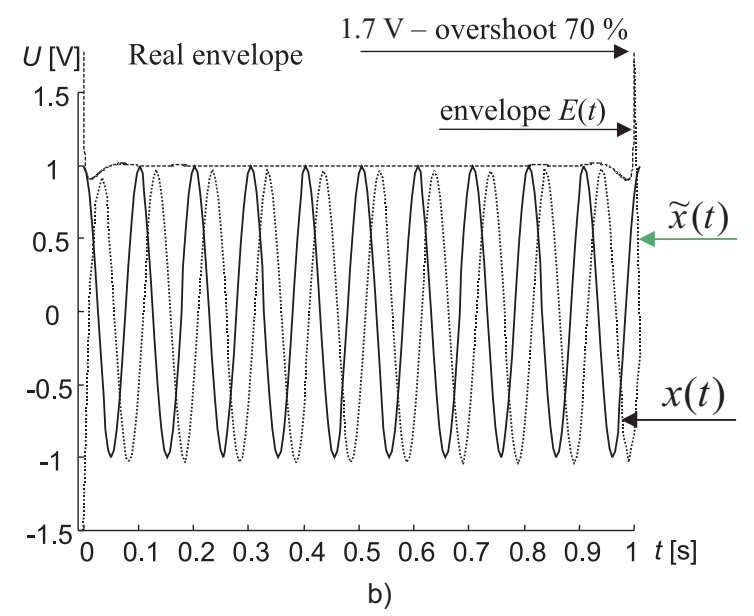

Fig. 2: Envelope distortion on the edge of the measurement interval, $x(t)=\sin (2 \pi 10 t+\varphi)$, sampling frequency $5 \mathrm{kHz}$ a) for $\varphi=0$, b) for $\varphi=\pi / 2$

\subsection{The step change effect on a harmonic signal envelope}

The envelope of harmonic signal modulated by a square pulse is shown in Figure 3a. The step is in the time when the harmonic signal is crossing zero (phase $\varphi_{\mathrm{sk}}=0$ ), and the quadrature part is numerically calculated. Figure $3 \mathrm{~b}$ shows the same signal but the step is at the time when the harmonic signal reaches the maximum (phase $\varphi_{\mathrm{sk}}=\pi / 2$ ).

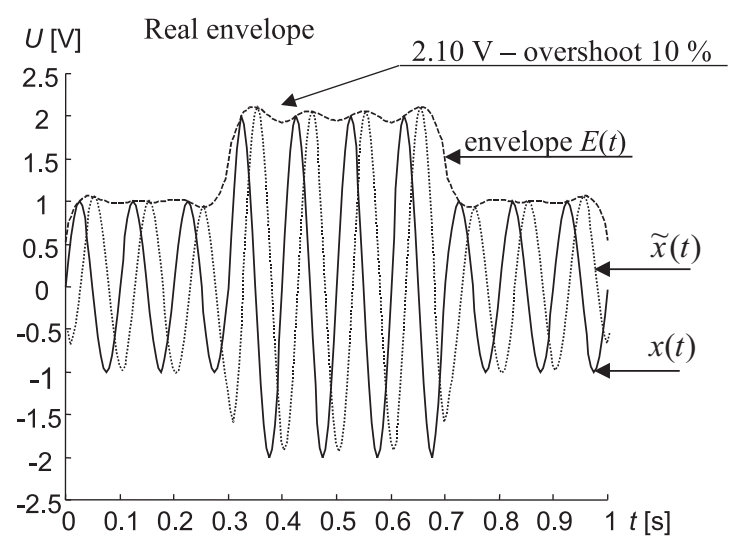

a)

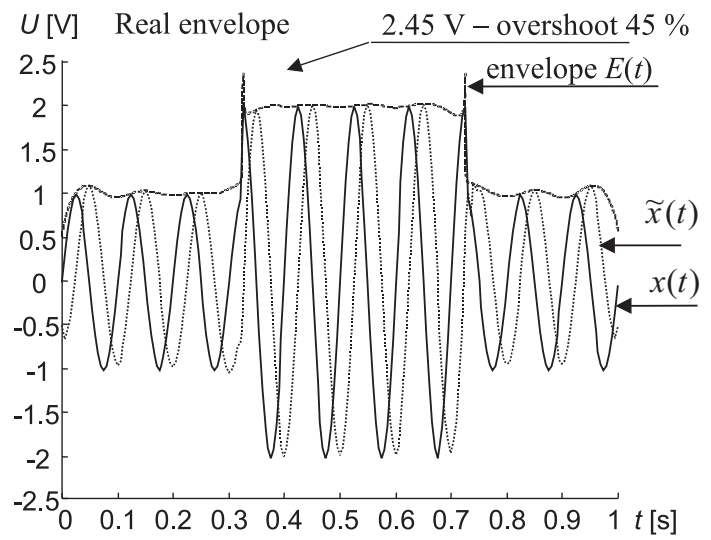

b)

Fig. 3: Envelope distortion on step change of harmonic signal, sampling frequency $5 \mathrm{kHz}, \varphi=0$

a) $x(t)=\sin (2 \pi 10 t+\varphi)[1+0.5(\operatorname{sign}(t-0.3)-\operatorname{sign}(t-0.7))]$

b) $x(t)=\sin (2 \pi 10 t+\varphi)[1+0.5(\operatorname{sign}(t-0.35)-\operatorname{sign}(t-0.75))]$

Figure 3 shows almost the same form of envelope as Figure 2. The overshoots produced by the step changes are smaller than the overshoot produced by the edge of the measurement interval.

\subsection{Envelope analysis errors recapitulation}

Figure 1 to Figure 3 show the different form of the envelope if it is calculated analytically or numerically at points of step change and at points of the edge of the measurement interval. The overshoot is theoretically infinite at the point of the step change (see Chapter 3.3). Calculation in discrete time only approximates this one. The signal in Figure $2 \mathrm{~b}$ has an overshoot of $70 \%$ of the original envelope. 
Figure 4 is a recapitulation of the systematic errors and a recapitulation of the theoretical values of the overshoots during the step change. The recapitulation is done for the harmonic signal $x(t)=E(t) \sin (2 \pi 10 t+\varphi)$ for the phase shift $\varphi=0$ and for phase shift $\varphi=\pi / 2$. The step change from $E(t)=0$ to $E(t)=1$ is in time $t=0$.

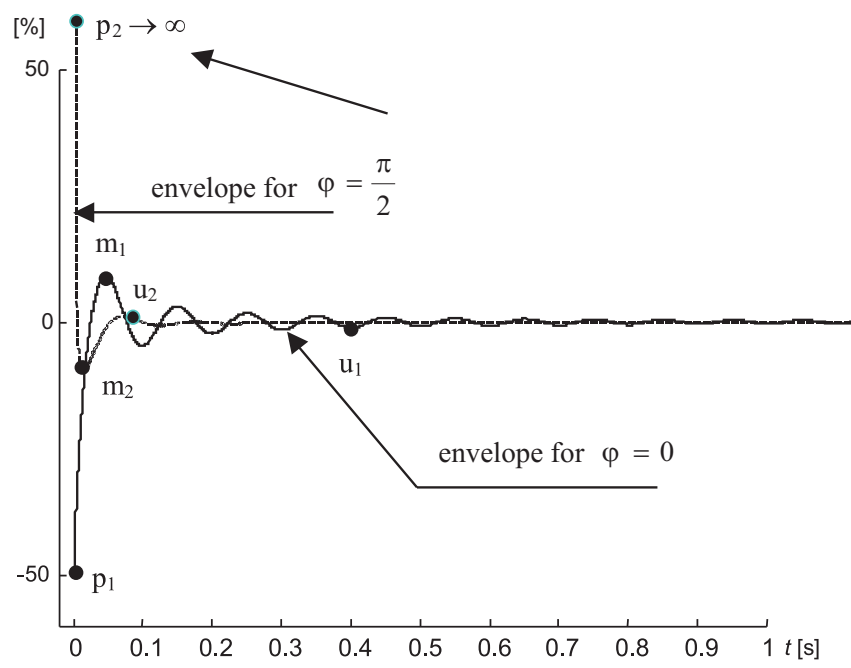

Fig. 4: Envelope error evaluation of harmonic signal for a step change in time $t=0$

The following lines show recapitulate the errors from Fig. 4:

a) for $\varphi=0$ and step change at time $t=0$ the envelope of signal $x(t)$ has:

-the first maximum overshoot (point $\left.\mathrm{p}_{1}\right) 50 \%$, at time $t=0 \mathrm{~ms}$

-the second maximum overshoot (point $\mathrm{m}_{1}$ ) $9.06 \%$, in time $t=0.045 \mathrm{~ms}$, i.e., 0.45 of the signal,

- stabilization (ripple $<1 \%$ ) (point $\mathrm{u}_{1}$ ), at time $t=0.405 \mathrm{~ms}$, i.e., 4.05 of the harmonic signal period.

b) for $\varphi=\pi / 2$ and step change at time $t=0$ the envelope of signal $x(t)$ has:

- the first maximum overshoot (point $\mathrm{p}_{2}$ ) $\infty$, at time $t=0 \mathrm{~ms}$,

- the second maximum overshoot (point $\mathrm{m}_{2}$ ) $9.44 \%$, at time $t=0.012 \mathrm{~ms}$, i.e., 0.12 of the signal period,

- stabilization (ripple $<1 \%$ ) (point $\mathrm{u}_{2}$ ), at time $t=0.080 \mathrm{~ms}$, i.e., 0.80 of the harmonic signal period.

An analytical calculation of the theoretical overshoot values of points $\mathrm{p}_{1}$ and $\mathrm{p}_{2}$ is given in Section 3.3.

\section{Analytical calculation of envelope overshoots}

This section gives a mathematical analysis of envelope behavior (parasitic overshoots) based on discovering the "non standard" behavior of the envelope (described in the previous chapter) calculated by the Hilbert transform in areas of step changes.

\subsection{Reason for envelope overshoots}

As was said in the introduction, the reason for these overshoots is the convolution function of the Hilbert transform $1 / \pi t$. This function for $t \rightarrow 0$ reaches as very high values, and for $t=0$ it is not defined. Thus it is necessary for the integral of the Hilbert transform

$$
\widetilde{x}(t)=\frac{1}{\pi} \int_{-\infty}^{\infty} \frac{x(\tau)}{t-\tau} \mathrm{d} \tau
$$

to be calculated as the values of the intrinsic integral according to the equation

$$
\widetilde{x}(t)=\lim _{\varepsilon \rightarrow-0} \frac{1}{\pi} \int_{-\infty}^{t-\varepsilon} \frac{x(\tau)}{t-\tau} \mathrm{d} \tau+\lim _{\varepsilon \rightarrow+0} \frac{1}{\pi} \int_{t-\varepsilon}^{\infty} \frac{x(\tau)}{t-\tau} \mathrm{d} \tau .
$$

The value of the integral is given by summation of limits. The limits calculated from the left and right side have a high difference when the calculation is made at the point of the step change. This is the source of the overshoots in the areas with step changes. The areas of step changes are also the beginning and the end of measurement signal.

\subsection{Overshoot amplitude calculation of a trapezoidal pulse}

Envelope overshoot behavior is mathematically analyzed on the basis of the example of the impulse combined from a trapezoidal and square pulse (further trapezoidal). This signal can simulate more types of signals by parameter changes. The trapezoidal signal is shown in Figure 5.

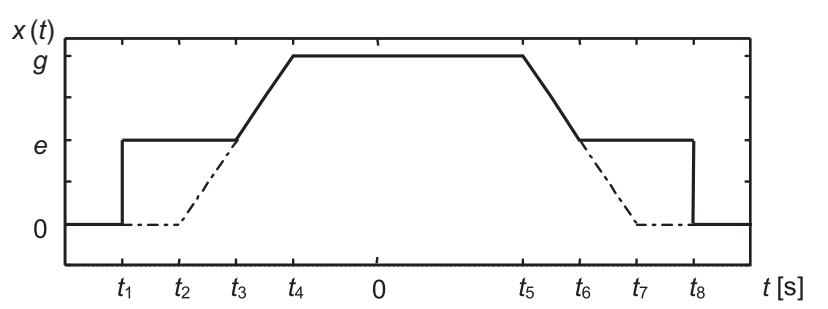

Fig. 5: The pulse combined from a trapezoidal and square pulse

The function shown in Figure 5 can be expressed as:

$$
x(t)= \begin{cases}0 & t \leq t_{1} \text { a } t \geq t_{8} \\ e & t_{1}<t<t_{3} \text { a } t_{6}<t<t_{8} \\ g & t_{4} \leq t \leq t_{5} \\ \frac{g\left(t-t_{2}\right)}{t_{4}-t_{2}} & t_{3} \leq t<t_{4} \\ -\frac{g\left(t-t_{7}\right)}{t_{7}-t_{5}} & t_{5}<t \leq t_{6}\end{cases}
$$

Using equation (13) for the quadrature part of trapezoidal pulse calculation

$$
\widetilde{x}(t)=\lim _{\varepsilon \rightarrow 0}-\frac{1}{\pi}\left(\int_{t_{1}+\varepsilon}^{t_{3}-\varepsilon} \frac{e}{\tau-t} \mathrm{~d} \tau+\frac{g}{t_{4}-t_{2}} \int_{t_{3}+\varepsilon}^{t_{4}-\varepsilon} \frac{\tau-t_{2}}{\tau-t} \mathrm{~d} \tau+\int_{t_{4}+\varepsilon}^{t_{5}-\varepsilon} \frac{g}{\tau-t} \mathrm{~d} \tau-\frac{g}{t_{7}-t_{5}} \int_{t_{5}+\varepsilon}^{t_{7}-\varepsilon} \frac{\tau-t_{7}}{\tau-t} \mathrm{~d} \tau+\int_{t_{6}+\varepsilon}^{t_{8}-\varepsilon} \frac{e}{\tau-t} \mathrm{~d} \tau\right)
$$




$$
\begin{aligned}
\widetilde{x}(t)=\lim _{\varepsilon \rightarrow 0}-\frac{1}{\pi} & \int_{t_{1}+\varepsilon}^{t_{3}-\varepsilon} \frac{e}{\tau-t} \mathrm{~d} \tau-\frac{g}{t_{4}-t_{2}} \int_{t_{3}+\varepsilon}^{t_{4}-\varepsilon} \frac{t_{2}}{\tau-t} \mathrm{~d} \tau+\frac{g}{t_{4}-t_{2}} \int_{t_{3}+\varepsilon}^{t_{4}-\varepsilon} \frac{\tau}{\tau-t} \mathrm{~d} \tau+ \\
& \left.+\int_{t 4+\varepsilon}^{t_{5}-\varepsilon} \frac{g}{\tau-t} \mathrm{~d} \tau-\frac{g}{t_{7}-t_{5}} \int_{t_{5}+\varepsilon}^{t_{6}-\varepsilon} \frac{\tau}{\tau-t} \mathrm{~d} \tau+\frac{g}{t_{7}-t_{5}} \int_{t_{5}+\varepsilon}^{t_{6}-\varepsilon} \frac{t_{7}}{\tau-t} \mathrm{~d} \tau+\int_{t_{6}+\varepsilon}^{t_{8}-\varepsilon} \frac{e}{\tau-t} \mathrm{~d} \tau\right) .
\end{aligned}
$$

Substituting for the integral yields (16)

$$
\begin{gathered}
\widetilde{x}(t)=-\frac{1}{\pi}\left\{e \ln \frac{\left|t_{3}-t\right|}{\left|t_{1}-t\right|}+e \ln \frac{\left|t_{8}-t\right|}{\left|t_{6}-t\right|}-\frac{g t_{2}}{t_{4}-t_{2}} \ln \frac{\left|t_{4}-t\right|}{\left|t_{3}-t\right|}+\frac{g t_{7}}{t_{7}-t_{5}} \ln \frac{\left|t_{6}-t\right|}{\left|t_{5}-t\right|}+g \ln \frac{\left|t_{5}-t\right|}{\left|t_{4}-t\right|}+\right. \\
\left.+\frac{g}{t_{4}-t_{2}}\left(t_{4}-t_{3}-t \ln \frac{\left|t_{4}-t\right|}{\left|t_{3}-t\right|}\right)-\frac{g}{t_{7}-t_{5}}\left(t_{7}-t_{5}-t \ln \frac{\left|t_{6}-t\right|}{\left|t_{5}-t\right|}\right)\right\} .
\end{gathered}
$$

Figure 6 illustrates the quadrature part $\widetilde{x}(t)$ and envelope $E(t)=\sqrt{x^{2}(t)+\widetilde{x}^{2}(t)}$ of signal $x(t)$ calculated by Equation (17), defined by Equation (14). Parameter $e=0$ was chosen to provide an easier view of the quadrature part and envelope.

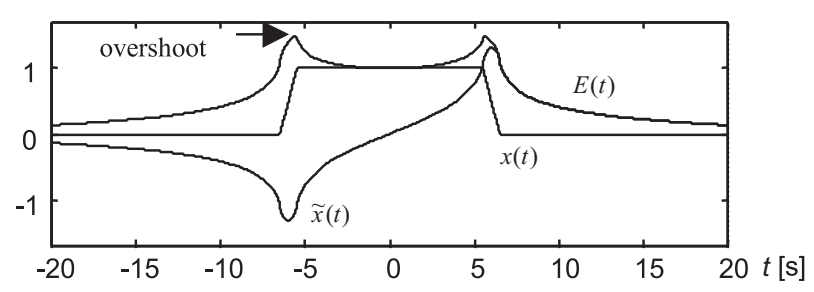

Fig. 6: Trapezoidal pulse given by (14), quadrature part $\widetilde{x}(t)$ and envelope $E(t)$ with parameters $e=0 ; g=1 ; t_{3}=-6.5 \mathrm{~s}$; $t_{4}=-5.5 \mathrm{~s} ; t_{5}=5.5 \mathrm{~s} ; t_{6}=6.5 \mathrm{~s} ; f_{\mathrm{vz}}=1 \mathrm{kHz}$

The dependency of the overshoot size (Figure 6) on the rising edge gradient is shown in Figure 7. This gradient is defined by parameter $s$. The dependency is determined numerically based, on any analytical expression of the quadrature part (17). The dependency cannot be determined analytically due to the complexity of Equation (17). Finding the local extreme by using the first derivative and then getting the overshoot dependency is very difficult.

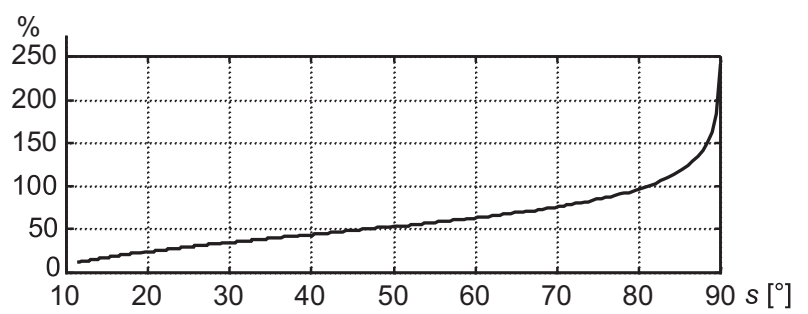

Fig. 7: Normalized graph of overshoot dependency on the gradient $s$ of a trapezoidal pulse rising edge.

Parameters $s=180$ atan $\left[(g-e) /\left(t_{6}-t_{5}\right)\right] / \pi$, where $e=0$, $g=1, t_{6}=6 \mathrm{~s}, t_{6}-t_{5} \in(0 \mathrm{~s}, 5 \mathrm{~s})$

It is evident from the graph that there is an overshoot amplitude of $50 \%$ for gradient $s=46,2^{\circ}$ of the rising edge. It is derived in [2] that the quadrature part of a square pulse (a trapezoidal pulse with a $90^{\circ}$ gradient) reaches an infinite value at this point. The difference between the theoretical amplitude $(\infty)$ and the amplitude given numerically (250\%, see Figure 7) is due to numerical calculation. Numerical calculation is not able to simulate a trapezoidal pulse with a $90^{\circ}$ gradient according to the finite distance of two following time samples. The maximum gradient that can be found by numerical calculation is given by

$$
s_{\max }=\frac{180}{\pi} \operatorname{atan}\left[(g-e) f_{\mathrm{vz}}\right]
$$

where $f_{\mathrm{vz}}$ is the sampling frequency.

It is possible to reach the gradient $s_{\max }=89.942^{\circ}$ for $f_{\mathrm{vz}}=1 \mathrm{kHz}$ and $g-e=1, s_{\max }=89.999^{\circ}$ for $f_{\mathrm{vz}}=1 \mathrm{MHz}$.

Further increasing of the sampling frequency only very slowly reaches the theoretical infinite amplitude.

\subsection{Overshoot amplitude calculation of a harmonic signal modulated by a trapezoidal pulse}

The following section describes the same analytical calculation as in the previous chapter, but for a harmonic signal modulated by the trapezoidal pulse shown in Figure 8 .

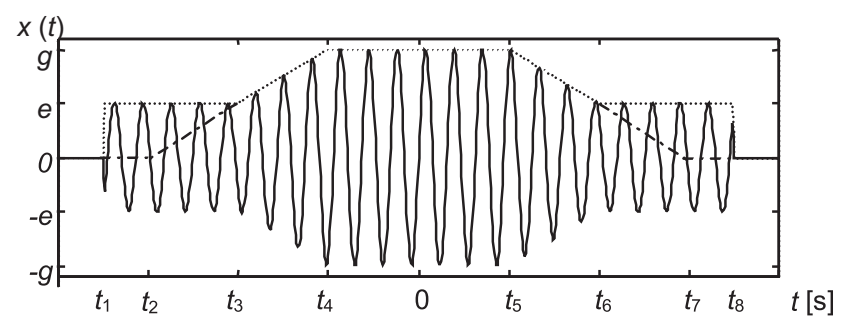

Fig. 8: Harmonic signal modulated by a trapezoidal pulse

The function in Figure 8 can be expressed as:

$$
x(t)= \begin{cases}0 & t \leq t_{1} \text { a } t \geq t_{8} \\ e \cos (\omega t+\varphi) & t_{1}<t<t_{3} \text { a } t_{6}<t<t_{8} \\ g \cos (\omega t+\varphi) & t_{4} \leq t \leq t_{5} \\ \frac{g\left(t-t_{2}\right)}{t_{4}-t_{2}} \cos (\omega t+\varphi) & t_{3} \leq t<t_{4} \\ -\frac{g\left(t-t_{7}\right)}{t_{7}-t_{5}} \cos (\omega t+\varphi) & t_{5}<t \leq t_{6}\end{cases}
$$




\subsubsection{Invariant part of a harmonic signal}

First we make a calculation of the invariant part of the harmonic signal, because it is easier to describe the calculation and because the Hilbert transform is linear according to addition. The invariant part of the harmonic signal modulated by the trapezoidal pulse of the signal in Fig. 8 is between points $t_{1}$ and $t_{2}$. The invariant part of the signal is shown in Fig. 9.

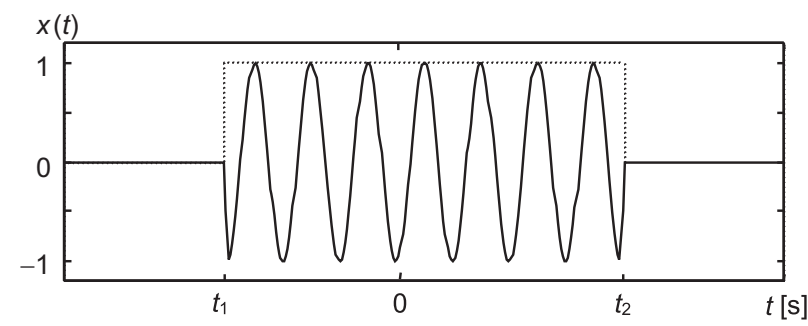

Fig. 9: Invariant part of the harmonic signal

The function shown in Fig. 9 can be expressed as:

$$
x(t)= \begin{cases}\cos \left(\omega_{0} t+\varphi\right) & \text { pro } t_{1}<t<t_{2}, \omega_{0}>0 \\ 0 & \text { pro } t<t_{1}, t>t_{2}\end{cases}
$$

The use of Equation (13) for calculating the quadrature part of function (20) yields

$$
\widetilde{x}(t)=-\frac{1}{\pi} \int_{t_{1}}^{t_{2}} \frac{\cos \left(\omega_{0} \tau+\varphi\right)}{\tau-t} \mathrm{~d} \tau .
$$

Substituting

$$
\begin{aligned}
& z=\tau-t \\
& \mathrm{~d} z=\mathrm{d} \tau \\
& z_{1}=t_{1}-t \\
& z_{2}=t_{2}-t
\end{aligned}
$$

Equation (21) can be written as:

$$
\widetilde{x}(t)=-\frac{1}{\pi} \int_{z_{1}}^{z_{2}} \frac{\cos \left(\omega_{0} z+\omega_{0} t+\varphi\right)}{z} \mathrm{~d} z .
$$

Equation (23) can be decomposed by equation (24) to equation (25)

$$
\cos (\alpha+\beta)=\cos (\alpha) \cos (\beta)-\sin (\alpha) \sin (\beta)
$$

and thus

$$
\tilde{x}(t)=-\frac{1}{\pi}\left[\cos \left(\omega_{0} t+\varphi\right) \int_{z_{1}}^{z_{2}} \frac{\cos \left(\omega_{0} z\right)}{z} \mathrm{~d} z-\sin \left(\omega_{0} t+\varphi\right) \int_{z_{1}}^{z_{2}} \frac{\sin \left(\omega_{0} z\right)}{z} \mathrm{~d} z\right] .
$$

The integral calculation inside Equation (25) has to be done separately according to a valid condition of integration [3]. The properties of odd and even functions, their integrals and integral sine and cosine are (27) in [2].

- for $t_{1}>t, z_{1}>0$ and for $t_{2}>t, z_{2}>0$

$$
\tilde{x}(t)=-\frac{1}{\pi}\left[\cos \left(\omega_{0} t+\varphi\right)\left(\int_{z_{1}}^{\infty} \frac{\cos \left(\omega_{0} z\right)}{z} \mathrm{~d} z-\int_{z_{2}}^{\infty} \frac{\cos \left(\omega_{0} z\right)}{z} \mathrm{~d} z\right)-\sin \left(\omega_{0} t+\varphi\right)\left(\int_{0}^{z_{2}} \frac{\sin \left(\omega_{0} z\right)}{z} \mathrm{~d} z-\int_{0}^{z_{1}} \frac{\sin \left(\omega_{0} z\right)}{z} \mathrm{~d} z\right)\right] .
$$

E; quation (26) changes by using inversion substitution (22) and substitution integral sine and cosine (27) to equation (30).

$$
\begin{aligned}
& C i(x)=-\int_{x}^{\infty} \frac{\cos (t)}{t} \mathrm{~d} t, \quad \operatorname{Si}(x)=\int_{0}^{x} \frac{\sin (t)}{t} \mathrm{~d} t \\
& \widetilde{x}(t)=-\frac{1}{\pi}\left[\cos \left(\omega_{0} t+\varphi\right)\left(-\operatorname{Ci}\left(\omega_{0} z_{1}\right)+\operatorname{Ci}\left(\omega_{0} z_{2}\right)\right)-\sin \left(\omega_{0} t+\varphi\right)\left(\operatorname{Si}\left(\omega_{0} z_{2}\right)-\operatorname{Si}\left(\omega_{0} z_{1}\right)\right)\right] \\
& \widetilde{x}(t)=\frac{1}{\pi}\left[\cos \left(\omega_{0} t+\varphi\right)\left(\operatorname{Ci}\left(\omega_{0} z_{1}\right)-\operatorname{Ci}\left(\omega_{0} z_{2}\right)\right)+\sin \left(\omega_{0} t+\varphi\right)\left(\operatorname{Si}\left(\omega_{0} z_{2}\right)-\operatorname{Si}\left(\omega_{0} z_{1}\right)\right)\right] \\
& \widetilde{x}(t)=\frac{1}{\pi}\left[\cos \left(\omega_{0} t+\varphi\right)\left(\operatorname{Ci}\left(\omega_{0}\left(t_{1}-t\right)\right)-\operatorname{Ci}\left(\omega_{0}\left(t_{2}-t\right)\right)\right)+\sin \left(\omega_{0} t+\varphi\right)\left(\operatorname{Si}\left(\omega_{0}\left(t_{2}-t\right)\right)-\operatorname{Si}\left(\omega_{0}\left(t_{1}-t\right)\right)\right)\right] .
\end{aligned}
$$

- for $t_{1}<t, z_{1}<0$ and for $t_{2}>t, z_{2}>0$

The extrinsic value of the integral has to be calculated on this condition, because the integral is not defined at zero. The function $\lim _{\varepsilon \rightarrow 0} f(x)$ will be written as $f(x)$ for easier notation. Simplification (31) will also be used further

$$
\begin{gathered}
C(z)=\frac{\cos \left(\omega_{0} z\right)}{z}, S(z)=\frac{\sin \left(\omega_{0} z\right)}{z} \\
\widetilde{x}(t)=-\frac{1}{\pi}\left\{\cos \left(\omega_{0} t+\varphi\right)\left(\int_{-\infty}^{0-\varepsilon} C(\mathrm{z}) \mathrm{d} z-\int_{-\infty}^{z_{1}} C(\mathrm{z}) \mathrm{d} z+\int_{0+\varepsilon}^{\infty} C(\mathrm{z}) \mathrm{d} z-\int_{z_{2}}^{\infty} C(\mathrm{z}) \mathrm{d} z\right)-\sin \left(\omega_{0} t+\varphi\right)\left(\int_{z_{1}}^{0-\varepsilon} S(\mathrm{z}) \mathrm{d} z+\int_{0+\varepsilon}^{z_{2}} S(\mathrm{z}) \mathrm{d} z\right)\right\}
\end{gathered}
$$


The Equation (33) and subsequently Equation (34) will be given by using the properties of even function $S(z)$ and odd function $C(z)$.

$\tilde{x}(t)=\lim _{\varepsilon \rightarrow 0}\left\{-\frac{1}{\pi}\left[\cos \left(\omega_{0} t+\varphi\right)\left(-\int_{-\infty}^{z_{1}} C(\mathrm{z}) \mathrm{d} z-\int_{z_{2}}^{\infty} C(\mathrm{z}) \mathrm{d} z\right)-\sin \left(\omega_{0} t+\varphi\right)\left(\int_{z_{1}}^{0-\varepsilon} S(\mathrm{z}) \mathrm{d} z+\int_{0+\varepsilon}^{z_{2}} S(\mathrm{z}) \mathrm{d} z\right)\right]\right\}$
$\widetilde{x}(t)=\lim _{\varepsilon \rightarrow 0}-\frac{1}{\pi}\left[\cos \left(\omega_{0} t+\varphi\right)\left(\int_{z_{1}}^{\infty} C(\mathrm{z}) \mathrm{d} z-\int_{z_{2}}^{\infty} C(\mathrm{z}) \mathrm{d} z\right)-\sin \left(\omega_{0} t+\varphi\right)\left(\int_{0+\varepsilon}^{-z_{1}} S(\mathrm{z}) \mathrm{d} z+\int_{0+\varepsilon}^{z_{2}} S(\mathrm{z}) \mathrm{d} z\right)\right]$

Equation (36) is given by conversion and by inversion substitution (22)

$\widetilde{x}(t)=-\frac{1}{\pi}\left[\cos \left(\omega_{0} t+\varphi\right)\left(-\operatorname{Ci}\left(\omega_{0} z_{1}\right)+\operatorname{Ci}\left(\omega_{0} z_{2}\right)\right)-\sin \left(\omega_{0} t+\varphi\right)\left(\operatorname{Si}\left(\omega_{0} z_{2}\right)+\operatorname{Si}\left(-\omega_{0} z_{1}\right)\right)\right]$

$\widetilde{x}(t)=\frac{1}{\pi}\left[\cos \left(\omega_{0} t+\varphi\right)\left(\operatorname{Ci}\left(\omega_{0}\left(t-t_{1}\right)\right)-\operatorname{Ci}\left(\omega_{0}\left(t_{2}-t\right)\right)\right)+\sin \left(\omega_{0} t+\varphi\right)\left(\operatorname{Si}\left(\omega_{0}\left(t_{2}-t\right)\right)+\operatorname{Si}\left(\omega_{0}\left(t-t_{1}\right)\right)\right)\right]$

- for $t_{1}<t, z_{1}<0$ and for $t_{2}<t, z_{2}<0$

$\widetilde{x}(t)=-\frac{1}{\pi}\left[\cos \left(\omega_{0} t+\varphi\right)\left(\int_{-\infty}^{z_{2}} C(\mathrm{z}) \mathrm{d} z-\int_{-\infty}^{z_{1}} C(\mathrm{z}) \mathrm{d} z\right)-\sin \left(\omega_{0} t+\varphi\right)\left(\int_{z_{1}}^{0} S(\mathrm{z}) \mathrm{d} z-\int_{z_{2}}^{0} S(\mathrm{z}) \mathrm{d} z\right)\right]$

Equation (38) will be given by using the properties of even function $S(z)$ and odd function $C(z)$.

$\tilde{x}(t)=-\frac{1}{\pi}\left[\cos \left(\omega_{0} t+\varphi\right)\left(\int_{-z_{1}}^{\infty} C(\mathrm{z}) \mathrm{d} z-\int_{-z_{2}}^{\infty} C(\mathrm{z}) \mathrm{d} z\right)-\sin \left(\omega_{0} t+\varphi\right)\left(\int_{0}^{-z_{1}} S(\mathrm{z}) \mathrm{d} z-\int_{0}^{-z_{2}} S(\mathrm{z}) \mathrm{d} z\right)\right]$

Equation (40) is given by conversion and inversion substitution.

$\tilde{x}(t)=-\frac{1}{\pi}\left[\operatorname{Cos}\left(\omega_{0} t+\varphi\right)\left(-\operatorname{Ci}\left(-\omega_{0} z_{1}\right)+\operatorname{Ci}\left(-\omega_{0} z_{2}\right)\right)-\sin \left(\omega_{0} t+\varphi\right)\left(\operatorname{Si}\left(-\omega_{0} z_{1}\right)-\operatorname{Si}\left(-\omega_{0} z_{2}\right)\right)\right]$

$\widetilde{x}(t)=\frac{1}{\pi}\left[\cos \left(\omega_{0} t+\varphi\right)\left(\operatorname{Ci}\left(\omega_{0}\left(t-t_{1}\right)\right)-\operatorname{Ci}\left(\omega_{0}\left(t-t_{2}\right)\right)\right)+\sin \left(\omega_{0} t+\varphi\right)\left(\operatorname{Si}\left(\omega_{0}\left(t-t_{1}\right)\right)-\operatorname{Si}\left(\omega_{0}\left(t-t_{2}\right)\right)\right)\right]$

Let us rewrite Equations (30), (36) and (40) as a function with parameters.

From Equation (30) it holds for $t<t_{1}<t_{2}$

$Y_{1}\left(t, t_{1}, t_{2}\right)=\frac{1}{\pi}\left[\cos \left(\omega_{0} t+\varphi\right)\left(\operatorname{Ci}\left(\omega_{0}\left(t_{1}-t\right)\right)-\operatorname{Ci}\left(\omega_{0}\left(t_{2}-t\right)\right)\right)+\sin \left(\omega_{0} t+\varphi\right)\left(\operatorname{Si}\left(\omega_{0}\left(t_{2}-t\right)\right)-\operatorname{Si}\left(\omega_{0}\left(t_{1}-t\right)\right)\right)\right]$

From Equation (36) it holds for $t_{1}<t<t_{2}$

$Y_{2}\left(t, t_{1}, t_{2}\right)=\frac{1}{\pi}\left[\cos \left(\omega_{0} t+\varphi\right)\left(\operatorname{Ci}\left(\omega_{0}\left(t-t_{1}\right)\right)-\operatorname{Ci}\left(\omega_{0}\left(t_{2}-t\right)\right)\right)+\sin \left(\omega_{0} t+\varphi\right)\left(\operatorname{Si}\left(\omega_{0}\left(t_{2}-t\right)\right)+\operatorname{Si}\left(\omega_{0}\left(t-t_{1}\right)\right)\right)\right]$

From equation (40) holds for $t_{1}<t_{2}<t$

$Y_{3}\left(t, t_{1}, t_{2}\right)=\frac{1}{\pi}\left[\cos \left(\omega_{0} t+\varphi\right)\left(\operatorname{Ci}\left(\omega_{0}\left(t-t_{1}\right)\right)-\operatorname{Ci}\left(\omega_{0}\left(t-t_{2}\right)\right)\right)+\sin \left(\omega_{0} t+\varphi\right)\left(\operatorname{Si}\left(\omega_{0}\left(t-t_{1}\right)\right)-\operatorname{Si}\left(\omega_{0}\left(t-t_{2}\right)\right)\right)\right]$

\subsubsection{Semifinite part of a harmonic signal}

A special case of the previous section is the semifinite part of the harmonic signal shown in Figure 10. as:

The function shown in the figure above can be expressed

$x(t)= \begin{cases}\cos \left(\omega_{0} t+\varphi\right) & \text { pro } t>0, \quad \omega_{0}>0 \\ 0 & \text { pro } t<0\end{cases}$

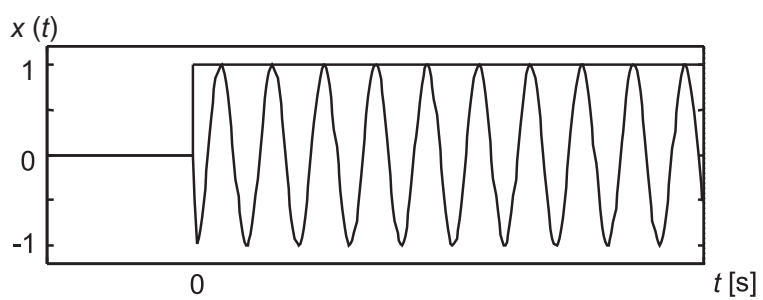

Fig. 10: Semifinite part of a harmonic signal 
The same result we obtained if we use the function defined by Equation (20), when $t_{1}=0, t_{2}=\infty$. Thus for Equation (30) and (36) it holds:

- for $t<0$

$$
\begin{aligned}
& \tilde{x}(t)=\frac{1}{\pi} \cos \left(\omega_{0} t+\varphi\right)\left[\operatorname{Ci}\left(-\omega_{0} t\right)\right]+ \\
& +\frac{1}{\pi} \sin \left(\omega_{0} t+\varphi\right)\left[\frac{\pi}{2}-\operatorname{Si}\left(-\omega_{0} t\right)\right]
\end{aligned}
$$

- for $t>0$

$$
\begin{aligned}
& \widetilde{x}(t)=\frac{1}{\pi} \cos \left(\omega_{0} t+\varphi\right)\left[\operatorname{Ci}\left(\omega_{0} t\right)\right]+ \\
& +\frac{1}{\pi} \sin \left(\omega_{0} t+\varphi\right)\left[\frac{\pi}{2}-\operatorname{Si}\left(-\omega_{0} t\right)\right]
\end{aligned}
$$

- for $t=0$ and $\varphi=\frac{\pi}{2}(2 k-1)$, where $k=1,2, \ldots$ is a positive integer, the value of $\widetilde{x}(t)$ according to Equation (47) is equal to \pm 0.5 .

$$
\widetilde{x}(0)=\frac{1}{2}(-1)^{k+1}
$$

For $t=0$ and $\varphi \neq \frac{\pi}{2}(2 k-1)$, and therefore for other phases except $\pi / 2,3 \pi / 2,5 \pi / 2, \ldots$ the quadrature signal is discontinuous, and it has an extrinsic limit of a logarithmic character [3] - see Equation (50).

$$
\begin{gathered}
\operatorname{Ci}(x)=-\int_{x}^{\infty} \frac{\cos (t)}{t} \mathrm{~d} t=\gamma+\ln x+\sum_{n=1}^{\infty}(-1)^{n} \frac{1}{2 n} \frac{x^{2 n}}{(2 n) !} \\
\text { where } \gamma=\lim _{n \rightarrow \infty}\left(1+\frac{1}{2}+\frac{1}{3}+\ldots+\frac{1}{n}-\ln (n)\right)=0.58 \\
\operatorname{Ci}(0)=-\int_{0}^{\infty} \frac{\cos (t)}{t} \mathrm{~d} t=\infty
\end{gathered}
$$

\subsubsection{Linearly rising part of a harmonic signal}

Let us make a calculation of the linearly rising part of harmonic signal modulated by a trapezoidal pulse between points $t_{3}$ and $t_{4}$, see Figure 8 . This part is shown in Figure 11, but points $t_{3}$ and $t_{4}$ are replaced by points $t_{1}$ and $t_{2}$.

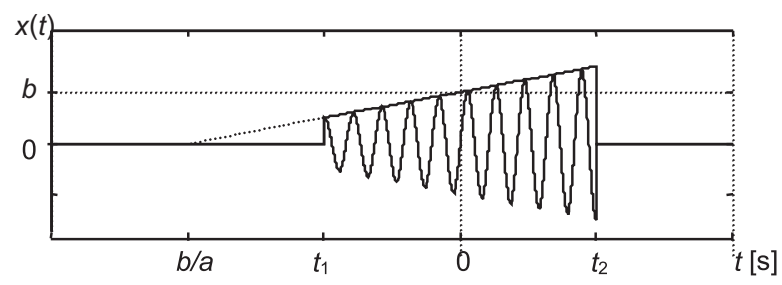

Fig. 11: Linearly rising part of a harmonic signal

The function shown in Figure 11 can be expressed as: $x(t)= \begin{cases}(a t+b) \cos \left(\omega_{0} t+\varphi\right) & \text { pro } t_{1}<t<t_{2}, \omega_{0}>0 \\ 0 & \text { pro } t<t_{1}, t>t_{2}\end{cases}$

Using Equation (13) for the quadrature part, the calculation of function (52) yields

$\tilde{x}(t)=-\frac{1}{\pi} \int_{t_{1}}^{t_{2}} \frac{(a \tau+b) \cos \left(\omega_{0} \tau+\varphi\right)}{\tau-t} \mathrm{~d} \tau$
$\tilde{x}(t)=-\frac{1}{\pi}\left(a \int_{t_{1}}^{t_{2}} \frac{\tau \cos \left(\omega_{0} \tau+\varphi\right)}{\tau-t} \mathrm{~d} \tau+b \int_{t_{1}}^{t_{2}} \frac{\cos \left(\omega_{0} \tau+\varphi\right)}{\tau-t} \mathrm{~d} \tau\right)$

Substituting

$$
\begin{aligned}
& z=\tau-t \\
& \mathrm{~d} z=\mathrm{d} \tau \\
& z_{1}=t_{1}-t \\
& z_{2}=t_{2}-t
\end{aligned}
$$

we can write Equation (53) as Equation (57)

$$
\begin{aligned}
& \tilde{x}(t)=-\frac{1}{\pi}\left[a \int_{z_{1}}^{z_{2}} \frac{(z+t) \cos \left(\omega_{0}(z+t)+\varphi\right)}{z} \mathrm{~d} z+b \int_{z_{1}}^{z_{2}} \frac{\cos \left(\omega_{0}(z+t)+\varphi\right)}{z} \mathrm{~d} z\right] \\
& \tilde{x}(t)=-\frac{1}{\pi}\left[a \int_{z_{1}}^{z_{2}} \cos \left(\omega_{0}(z+t)+\varphi\right) \mathrm{d} z+a t \int_{z_{1}}^{z_{2}} \frac{\cos \left(\omega_{0}(z+t)+\varphi\right)}{z} \mathrm{~d} z+b \int_{z_{1}}^{z_{2}} \frac{\cos \left(\omega_{0}(z+t)+\varphi\right)}{z} \mathrm{~d} z\right] \\
& \tilde{x}(t)=-\frac{1}{\pi}\left[\int_{z_{1}}^{z_{2}} \cos \left(\omega_{0}(z+t)+\varphi\right) \mathrm{d} z+(a t+b) \int_{z_{1}}^{z_{2}} \frac{\cos \left(\omega_{0}(z+t)+\varphi\right)}{z} \mathrm{~d} z\right]
\end{aligned}
$$

Let us make a separate calculation of the integral using (58) in Equation (57)

$$
\widetilde{x}(t)=-\frac{1}{\pi}\left[I_{1}(t)+I_{2}(t)\right]
$$

where there is $I_{1}(t)=a \int_{z_{1}}^{z_{2}} \cos \left(\omega_{0}(z+t)+\varphi\right) \mathrm{d} z$ 


$$
I_{2}(t)=(a t+b) \int_{z_{1}}^{z_{2}} \frac{\cos \left(\omega_{0}(z+t)+\varphi\right)}{z} \mathrm{~d} z
$$

For calculation of $I_{1}(t)$ it holds:

$$
I_{1}(t)=\frac{a}{\omega_{0}}\left[\sin \left(\omega_{0}\left(z_{2}+t\right)+\varphi\right)-\sin \left(\omega_{0}\left(z_{1}+t\right)+\varphi\right)\right]
$$

by using inverse substitution (54) we have

$$
I_{1}(t)=\frac{a}{\omega_{0}}\left[\sin \left(\omega_{0} t_{2}+\varphi\right)-\sin \left(\omega_{0} t_{1}+\varphi\right)\right]
$$

The result of Equations (30), (36) and (40) will be used for calculating of $I_{2}(t)$.

- for $t<t_{1}<t_{2}$

$I_{2 a}(t)=(a t+b)\left\{\operatorname{Cos}\left(\omega_{0} t+\varphi\right)\left[\operatorname{Ci}\left(\omega_{0}\left(t_{1}-t\right)\right)-\operatorname{Ci}\left(\omega_{0}\left(t_{2}-t\right)\right)\right]+\sin \left(\omega_{0} t+\varphi\right)\left[\operatorname{Si}\left(\omega_{0}\left(t_{2}-t\right)\right)-\operatorname{Si}\left(\omega_{0}\left(t_{1}-t\right)\right)\right]\right\}$

- for $t_{1}<t<t_{2}$

$I_{2 b}(t)=(a t+b)\left\{\operatorname{Cos}\left(\omega_{0} t+\varphi\right)\left[\operatorname{Ci}\left(\omega_{0}\left(t-t_{1}\right)\right)-\operatorname{Ci}\left(\omega_{0}\left(t_{2}-t\right)\right)\right]+\sin \left(\omega_{0} t+\varphi\right)\left[\operatorname{Si}\left(\omega_{0}\left(t_{2}-t\right)\right)+\operatorname{Si}\left(\omega_{0}\left(t-t_{1}\right)\right)\right]\right\}$

- for $t_{1}<t_{2}<t$

$I_{2 c}(t)=(a t+b)\left\{\operatorname{Cos}\left(\omega_{0} t+\varphi\right)\left[\operatorname{Ci}\left(\omega_{0}\left(t-t_{1}\right)\right)-\operatorname{Ci}\left(\omega_{0}\left(t-t_{2}\right)\right)\right]+\sin \left(\omega_{0} t+\varphi\right)\left[\operatorname{Si}\left(\omega_{0}\left(t-t_{1}\right)\right)-\operatorname{Si}\left(\omega_{0}\left(t-t_{2}\right)\right)\right]\right\}$

Let us rewrite the Equations (62), (63), (64) and (65) as a function with parameters.

For Equation (62), (63) and for $t<t_{1}<t_{2}$ it holds:

$Y_{4}\left(t, t_{1}, t_{2}, a, b\right)=-\frac{1}{\pi}\left[I_{1}\left(t, t_{1}, t_{2}, a\right)+I_{2 a}\left(t, t_{1}, t_{2}, a, b\right)\right]$

For Equation (62), (64) and for $t_{1}<t<t_{2}$ it holds

$Y_{5}\left(t, t_{1}, t_{2}, a, b\right)=-\frac{1}{\pi}\left[I_{1}\left(t, t_{1}, t_{2}, a\right)+I_{2 b}\left(t, t_{1}, t_{2}, a, b\right)\right]$

For equation (62), (65) and for $t_{1}<t_{2}<t$

$Y_{6}\left(t, t_{1}, t_{2}, a, b\right)=-\frac{1}{\pi}\left[I_{1}\left(t, t_{1}, t_{2}, a\right)+I_{2 c}\left(t, t_{1}, t_{2}, a, b\right)\right]$

\subsubsection{Total analytical calculation of the quadrature part of a harmonic signal modulated by trapezoidal pulse}

As was mentioned above, it was necessary to make the calculation of the quadrature part of a harmonic signal modulated by a trapezoidal pulse in separate parts, due to the complexity of the analytical calculation.

Now we will make a summary of all the parts, see Figure 8. We obtain the entire quadrature signal $\widetilde{x}(t)$ by the sum of all parts with respect to important parameters in the individual part.

- for $t \leq t_{1}$

$$
Z_{1}(t)=Y_{1}\left(t, t_{1}, t_{3}\right)+Y_{4}\left(t, t_{3}, t_{4}, \frac{g-e}{t_{4}-t_{3}}, \frac{e t_{4}-g t_{3}}{t_{4}-t_{3}}\right)+Y_{1}\left(t, t_{4}, t_{5}\right)+Y_{4}\left(t, t_{5}, t_{6}, \frac{e-g}{t_{6}-t_{5}}, \frac{g t_{6}-e t_{5}}{t_{6}-t_{5}}\right)+Y_{1}\left(t, t_{6}, t_{8}\right)
$$

- for $t_{1}<t<t_{3}$

$$
Z_{2}(t)=Y_{2}\left(t, t_{1}, t_{3}\right)+Y_{4}\left(t, t_{3}, t_{4}, \frac{g-e}{t_{4}-t_{3}}, \frac{e t_{4}-g t_{3}}{t_{4}-t_{3}}\right)+Y_{1}\left(t, t_{4}, t_{5}\right)+Y_{4}\left(t, t_{5}, t_{6}, \frac{e-g}{t_{6}-t_{5}}, \frac{g t_{6}-e t_{5}}{t_{6}-t_{5}}\right)+Y_{1}\left(t, t_{6}, t_{8}\right)
$$

- for $t_{3} \leq t<t_{4}$

$$
Z_{3}(t)=Y_{3}\left(t, t_{1}, t_{3}\right)+Y_{5}\left(t, t_{3}, t_{4}, \frac{g-e}{t_{4}-t_{3}}, \frac{e t_{4}-g t_{3}}{t_{4}-t_{3}}\right)+Y_{1}\left(t, t_{4}, t_{5}\right)+Y_{4}\left(t, t_{5}, t_{6}, \frac{e-g}{t_{6}-t_{5}}, \frac{g t_{6}-e t_{5}}{t_{6}-t_{5}}\right)+Y_{1}\left(t, t_{6}, t_{8}\right)
$$

- for $t_{4} \leq t \leq t_{5}$

$$
Z_{4}(t)=Y_{3}\left(t, t_{1}, t_{3}\right)+Y_{6}\left(t, t_{3}, t_{4}, \frac{g-e}{t_{4}-t_{3}}, \frac{e t_{4}-g t_{3}}{t_{4}-t_{3}}\right)+Y_{2}\left(t, t_{4}, t_{5}\right)+Y_{4}\left(t, t_{5}, t_{6}, \frac{e-g}{t_{6}-t_{5}}, \frac{g t_{6}-e t_{5}}{t_{6}-t_{5}}\right)+Y_{1}\left(t, t_{6}, t_{8}\right)
$$

- for $t_{5}<t \leq t_{6}$

$$
Z_{5}(t)=Y_{3}\left(t, t_{1}, t_{3}\right)+Y_{6}\left(t, t_{3}, t_{4}, \frac{g-e}{t_{4}-t_{3}}, \frac{e t_{4}-g t_{3}}{t_{4}-t_{3}}\right)+Y_{3}\left(t, t_{4}, t_{5}\right)+Y_{5}\left(t, t_{5}, t_{6}, \frac{e-g}{t_{6}-t_{5}}, \frac{g t_{6}-e t_{5}}{t_{6}-t_{5}}\right)+Y_{1}\left(t, t_{6}, t_{8}\right)
$$

- for $t_{6}<t<t_{8}$

$$
Z_{6}(t)=Y_{3}\left(t, t_{1}, t_{3}\right)+Y_{6}\left(t, t_{3}, t_{4}, \frac{g-e}{t_{4}-t_{3}}, \frac{e t_{4}-g t_{3}}{t_{4}-t_{3}}\right)+Y_{3}\left(t, t_{4}, t_{5}\right)+Y_{6}\left(t, t_{5}, t_{6}, \frac{e-g}{t_{6}-t_{5}}, \frac{g t_{6}-e t_{5}}{t_{6}-t_{5}}\right)+Y_{2}\left(t, t_{6}, t_{8}\right)
$$

- for $t_{8}<t$

$$
Z_{7}(t)=Y_{3}\left(t, t_{1}, t_{3}\right)+Y_{6}\left(t, t_{3}, t_{4}, \frac{g-e}{t_{4}-t_{3}}, \frac{e t_{4}-g t_{3}}{t_{4}-t_{3}}\right)+Y_{3}\left(t, t_{4}, t_{5}\right)+Y_{6}\left(t, t_{5}, t_{6}, \frac{e-g}{t_{6}-t_{5}}, \frac{g t_{6}-e t_{5}}{t_{6}-t_{5}}\right)+Y_{3}\left(t, t_{6}, t_{8}\right)
$$


The entire equation for the quadrature part is done by the sum of Equations (69)-(75):

$\tilde{x}(t)=Z_{1}(t)+Z_{2}(t)+Z_{3}(t)+Z_{4}(t)+Z_{5}(t)+Z_{6}(t)+Z_{7}(t)$

The quadrature part $\widetilde{x}(t)$ calculated by (76) and the envelope $E(t)=\sqrt{x^{2}(t)+\widetilde{x}^{2}(t)}$ of signal $x(t)$ defined by Equation (19) is shown in Figure 12.

The dependency of the overshoot size (Fig. 12) on the rising edge gradient is shown in Figure 13. This gradient is defined by parameter $s$. The dependency is determined numerically based on an analytical expression of the quadrature part (76). The dependency cannot be determined analytically due to the complexity of Equation (76). Finding the local extreme by using the first derivative and then getting the overshoot dependency is very difficult.

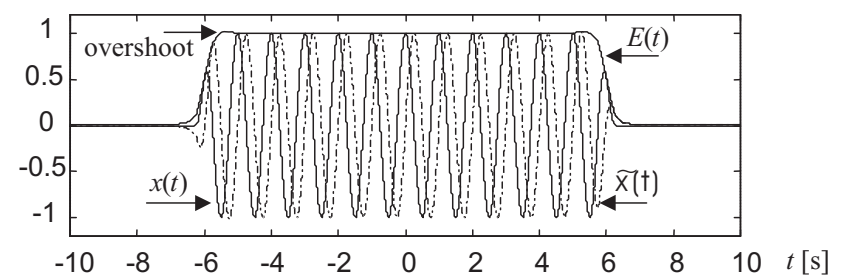

Fig. 12: Harmonic signal modulated by trapezoidal pulse $x(t)$ given (19), quadrature part $\widetilde{x}(t)$ and envelope $E(t)$ with parameters $e=0 ; g=1 ; t_{3}=-6.25 \mathrm{~s} ; t_{4}=-5.75 \mathrm{~s}$; $t_{5}=5.75 \mathrm{~s} ; t_{6}=6.25 \mathrm{~s} ; \omega_{0}=2 \pi ; \varphi=0, \mathrm{f}_{\mathrm{vz}}=1 \mathrm{kHz}$

Notice: Parameters $t_{1}$ and $t_{8}$ lose their relevance if the parameter $e=0$. For this reason these parameters were not mentioned. Parameter $e=0$ was chosen to provide an easier view of the quadrature part and envelope.

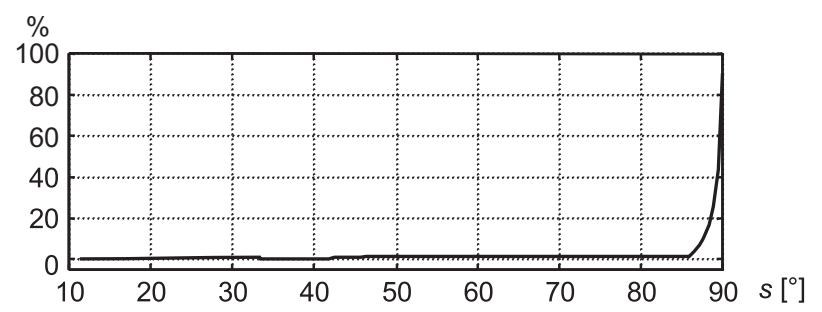

Fig. 13: Normalized graph of overshoot dependency on the gradient $s$ of the signal (19) rising edge, parameters $s=180 \operatorname{atan}\left[(g-e) /\left(t_{6}-t_{5}\right)\right] / \pi$, where $e=0, g=1$, $t_{6}=6 \mathrm{~s}, t_{6}-t_{5} \in(0 \mathrm{~s}, 5 \mathrm{~s}), \omega_{0}=2 \pi, \varphi=0$

It is evident from the graph that there is an overshoot amplitude of $50 \%$ for gradient $s=89.5^{\circ}$ of the rising edge. It is derived in [2], that the quadrature part of a harmonic signal modulated by a square pulse (a trapezoidal pulse with a $90^{\circ}$ gradient) reaches an infinite value at this point. It is also evident that there is a high amplitude rise for gradient $s>86^{\circ}$. It was derived in Equations $(44)-(50)$ that the quadrature part of the harmonic signal $\cos \left(\omega_{0} t+\varphi\right)$ reaches an infinite amplitude at any point with a step change and when the relation $\cos \left(\omega_{0} t+\varphi\right) \neq 0$ is valid.

The difference between the theoretical amplitude $(\infty)$ and the amplitude given numerically (90\%, see Figure 13) is due to numerical calculation. Numerical calculation is not able to simulate a trapezoidal pulse with a $90^{\circ}$ gradient according to the finite distance of two following time samples. The maximum gradient that can be reached by numerical calculation is given by

$$
s_{\max }=\frac{180}{\pi} \operatorname{atan}\left((g-e) f_{\mathrm{vz}}\right)
$$

where $f_{\mathrm{vz}}$ is the sampling frequency.

It is possible to reach the gradient $s_{\max }=89.936^{\circ}$ for $f_{\mathrm{vz}}=1 \mathrm{kHz}$ and $\mathrm{g}-\mathrm{e}=1, s_{\max }=89.999^{\circ}$ for $f_{\mathrm{vz}}=1 \mathrm{MHz}$.

A further increase in sampling frequency only very slowly approaches to the theoretical amplitude.

\section{Conclusion}

The dependency of the size overshoot on the gradient edge of a pulse was found. This was done by an analytical calculation of the quadrature part of a trapezoidal pulse and of a harmonic signal modulated by a trapezoidal pulse. The same source of the envelope size overshoot is the gradient edge of a pulse and also that beginning and end of the measurement interval. The size of the overshoot of a harmonic signal modulated by a trapezoidal pulse is $80 \%$ with a gradient of edge almost $90^{\circ}$. The size of the overshoot of the trapezoidal pulse is $250 \%$. The analytical dependency of the size of the overshoot as not found, due to mathematical complexity. Only numerical calculation was done, based on previous analytical calculation of the quadrature part.

\section{Acknowledgement}

This research work has received support from research program No J04/98:210000015 "Research of New Methods for Physical Quantities Measurement and Their Application in Instrumentation" of the Czech Technical University in Prague (sponsored by the Ministry of Education, Youth and Sports of the Czech Republic).

\section{References}

[1] Hahn, S. L.: Hilbert Transforms in Signal Processing. Artech House, Boston, 1966

[2] Doubek, J.: Integrálni transformace ve zpracováni vibrodiagnostického signálu. Dizertační práce, ČVUT Fakulta elektrotechnická, 2001, (In Czech: Integral Transform in a Vibrodiagnostic Signal), Ph.D. thesis, FEE, CTU, Prague, 2001

[3] Čížek, V.: Discrete Hilbert Transform. IEEE Transaction on Audio and Electronic, Volume AU-18, No. 4, December 1970, pp. 340-342

[4] Doubek, J., Kreidl, M.: New Algorithms of Gearbox Faults Detection Using Hilbert Transform and Cross Corelation. Workshop, CTU Publishing House, Prague, 2001, pp. 320-321

[5] Kreidl, M., et al: Diagnostické systémy. Monografie, Ediční středisko ČVUT, Praha, (In Czech: Diagnostic Systems). Monograph, FEE CUT, Prague, 2001

Doc. Ing. Marcel Kreidl, CSc.

e-mail: kreidl@fel.cvut.cz

Ing. Jan Doubek, Ph.D.

e-mail: doubek@fel.cvut.cz

Department of Measurement

Czech Technical University in Prague

Faculty of Electrical Engineering

Technická 2, 16627 Praha 6, Czech Republic 